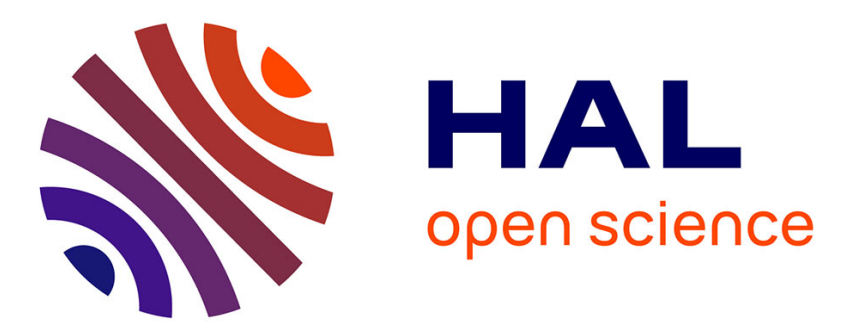

\title{
Positivity conditions of Lyapunov functions for systems with slope restricted nonlinearities
}

Giorgio Valmorbida, R. Drummond, S. Duncan

\section{To cite this version:}

Giorgio Valmorbida, R. Drummond, S. Duncan. Positivity conditions of Lyapunov functions for systems with slope restricted nonlinearities. 2016 American Control Conference (ACC), Jul 2016, Boston, United States. 10.1109/ACC.2016.7524925 . hal-01710297

\section{HAL Id: hal-01710297 https://hal.science/hal-01710297}

Submitted on 13 Apr 2020

HAL is a multi-disciplinary open access archive for the deposit and dissemination of scientific research documents, whether they are published or not. The documents may come from teaching and research institutions in France or abroad, or from public or private research centers.
L'archive ouverte pluridisciplinaire HAL, est destinée au dépôt et à la diffusion de documents scientifiques de niveau recherche, publiés ou non, émanant des établissements d'enseignement et de recherche français ou étrangers, des laboratoires publics ou privés. 


\title{
Positivity Conditions of Lyapunov Functions for Systems with Slope Restricted Nonlinearities
}

\author{
G. Valmorbida, R. Drummond, S. R. Duncan
}

\begin{abstract}
This paper considers absolute stability for Lur'e systems consisting of the interconnection of a linear plant with a nonlinear feedback. The nonlinearity is assumed to be both sector bounded and slope restricted. Stability of this system is determined using a Lyapunov function with a quadratic term on both the states and the nonlinearity. The main result of this paper is to relax the positivity conditions that have been imposed for such Lyapunov functions. This allows Lyapunov functions to be constructed without a positive definite quadratic matrix and whose scalars of the Lur'e term are not sign-definite. We also show that previous results can be simplified to the case of the quadratic form with Lur'e term. The benefits of considering such a Lyapunov function for stability analysis are shown both for the global case and for the local case.
\end{abstract}

Index Terms - Nonlinear systems, Lyapunov analysis, positivity conditions

\section{INTRODUCTION}

This paper is concerned with the absolute stability of linear time-invariant systems with slope-restricted input nonlinearities. Absolute stability is a classical problem in systems and control theory that has been long studied using frequency based methods including the celebrated circle and Popov criterions for sector bounded nonlinearities [10]. These methods rely on the representation of the nonlinear system via the interconnection of a linear term and a static nonlinearity, which allows the analysis of the loop to be performed by assessing the properties of the linear component. Variants of these methods for the cases of MIMO systems [16] and multiple equilibria [11] have also been developed. A strong motivation for studying the class of slope-restricted nonlinearities was set by the Kalman Conjecture [9].

Developments of these frequency based methods have been proposed to reduce the conservatism on the analysis. The introduction of multipliers into the stability problem, with the most famous example being the Zames-Falb multiplier, has been shown to improve results

G. Valmorbida, is with Laboratoire des Signaux et Systèmes, CentraleSupélec, CNRS, Univ. Paris-Sud, Université ParisSaclay, 3 Rue Joliot-Curie, Gif-sur-Yvette 91192, France giorgio.valmorbida@12s.centralesupelec.fr .R. Drummond and S. R. Duncan are with the Control Group, Department of Engineering Science, University of Oxford, 17 Parks Road, OX1 3PJ Oxford, United Kingdom \{ross.drummond, stephen.duncan\}@eng.ox.ac.uk. Work supported in part by EPSRC grants EP/J010537/1 and EP/L505031/1. at the expense of computational complexity (see [3] for recent a review on the contribution of the works of O'Shea [13] and Zames \& Falb [20]). In recent years, methods have been proposed to reduce the complexity of multipliers [3]. Composite Lyapunov functions (LF), where the LFs are constructed from the convex hull of a set of invariant ellipsoids, have also been proposed [7]. LFs with the quadratic components with respect to both the nonlinearities and the states and Lur'e terms were studied in $[15,18]$. Many of the tools and methods for absolute stability analysis can be framed within the integral quadratic constraint framework which generalises the frequency based approach as originally studied with the circle and Popov criterions [12].

For quadratic LFs, the positivity of the LF is enforced by requiring a quadratic matrix to be positivedefinite [10]. In the case of Lur'e-type LFs, the relaxation of the positivity constraints in the Lur'e coefficients has been studied in $[6,14]$.

\section{A. Contribution}

This paper will further develop LFs that are quadratic on both the state and the nonlinear terms and also contain a Lur'e term. We present conditions for the positivity of the LF that do not impose the positivity of the scalars of the Lur'e terms nor impose the quadratic terms on the nonlinearities to be positive definite.

The conditions for the positivity of both the LF and its time-derivative rely on sector conditions that express the fact that both the nonlinearities lie in a sector and their slopes are bounded. By allowing the sector condition to hold only locally (in a compact set containing the origin), we can formulate conditions for the local stability analysis and obtain estimates of the region of attraction with contractive and invariant sets defined by level sets of the LF. The proposed results are applied to a system containing a logarithmic nonlinearity which satisfies a sector condition only locally.

We also show that a number of Lur'e terms for slope restricted nonlinearities presented in [18] yield the proposed structure with a quadratic term on both the state and the nonlinearities and a single Lur'e term.

Notation The set of real valued matrices of dimensions $n \times m$ is denoted $\mathbb{R}^{n \times m}$, the set of symmetric matrices of dimension $n$ is denoted $\mathbb{S}^{n}$, the set of diagonal matrices is denoted $\mathbb{D}$, the set of positive semi-definite 
diagonal matrices is denoted $\mathbb{D}_{\geq 0}$. The interior of a set $\mathcal{D} \subset \mathbb{R}^{m}$ is denoted $\mathcal{D}^{\circ}, I_{m}$ denotes the identity matrix of dimension $m, 1_{m}$ denotes the row vector of dimension $m$ of which the entries are equal to 1 . With $\alpha \geq 0$ we use $\mathcal{E}(V, \alpha)=\left\{x \in \mathbb{R}^{n} \mid V(x) \geq \alpha\right\}$ i.e. the $\alpha$ sublevel set of a positive-definite function $V$. We may drop the arguments of some functions when it is clear from the context.

\section{Problem Statement}

Consider the linear time-invariant (LTI) system with input nonlinearities

$$
\left\{\begin{array}{l}
\dot{x}=A x+B \phi(y) \\
y=C x+D \phi(y)
\end{array}\right.
$$

with $x \in \mathbb{R}^{n}, y \in \mathbb{R}^{m}$. The nonlinearity $\phi: \mathcal{Y} \rightarrow \mathbb{R}^{m}$, $\mathcal{Y} \subseteq \mathbb{R}^{m}$ is assumed time-invariant, Lipschitz on $\mathcal{Y}^{\circ}$, decentralized

$$
\phi(y)=\left[\begin{array}{lll}
\phi_{1}\left(y_{1}\right) & \phi_{2}\left(y_{2}\right) & \ldots \phi_{m}\left(y_{m}\right)
\end{array}\right]^{T},
$$

sector bounded

$$
\frac{\phi_{i}\left(y_{i}\right)}{y_{i}} \in\left[\underline{\delta_{i}}, \overline{\delta_{i}}\right] \quad \forall y \in \mathcal{Y}_{0} \subseteq \mathcal{Y}
$$

which implies $\phi(0)=0$, and slope restricted

$$
\partial \phi_{i}\left(y_{i}\right) \in\left[\underline{\gamma}_{i}, \bar{\gamma}_{i}\right] \quad \forall y \in \mathcal{Y}_{0} \subseteq \mathcal{Y}
$$

where $\partial$ denotes the sub-differential operator. The Lipschitz assumption on $\phi$ implies that $\partial \phi_{i}\left(y_{i}\right)=\frac{d \phi_{i}}{y_{i}}$ almost everywhere, relaxing the requirement for the nonlinearity to be continuously differentiable [18, Section 2]. Via loop transformations that modify both the linear component and the nonlinearity, a sector non-linearity which lies in the sector $\left[\underline{\delta_{i}}, \overline{\delta_{i}}\right]$ can be transformed into a sector nonlinearity that lies in the sector $[0,1]$ (see $[10$, page 233$]$ ). However, in this paper we preserve the sector $\left[\underline{\delta_{i}}, \overline{\delta_{i}}\right]$ to study stability properties of system (1). Figures $1-2$ illustrate the above definitions. We also introduce the matrices $\underline{\Delta}:=\operatorname{diag}\left(\underline{\delta_{1}}, \ldots, \underline{\delta_{m}}\right), \bar{\Delta}:=\operatorname{diag}\left(\overline{\delta_{1}}, \ldots, \overline{\delta_{m}}\right)$ $\underline{\Gamma}:=\operatorname{diag}\left(\underline{\gamma_{1}}, \ldots, \underline{\gamma_{m}}\right), \bar{\Gamma}:=\operatorname{diag}\left(\overline{\gamma_{1}}, \ldots, \overline{\gamma_{m}}\right)$ to compactly express the sector and slope bounds.

Assumption 2.1 (Well-posedness): The algebraic loop in (1) is well-posed for all $x \in \mathcal{X}, 0 \in \mathcal{X}$. That is, for each $x \in \mathcal{X} \subseteq \mathbb{R}^{n}$ it corresponds a unique $y$ satisfying the equation $y-D \phi(y)=C x$. Hence the mapping $y: \mathcal{X} \rightarrow \mathcal{Y}$ is well-defined.

Provided the above assumption holds, we can define the following set

$$
\mathcal{X}_{0}:=\left\{x \in \mathbb{R}^{n} \mid y(x) \in \mathcal{Y}_{0}\right\},
$$

where the set $\mathcal{Y}_{0} \subset \mathbb{R}^{m}$ corresponds to the set where the sector and the slope restrictions hold, as defined in (2). Under Assumption 2.1 this paper proposes a solution to the following problem:

Problem 2.1: For system (1) with slope restricted nonlinearity $\phi$, find a Lyapunov function to certify the stability of the origin with an estimate of the region of attraction contained in $\mathcal{X}_{0}$.

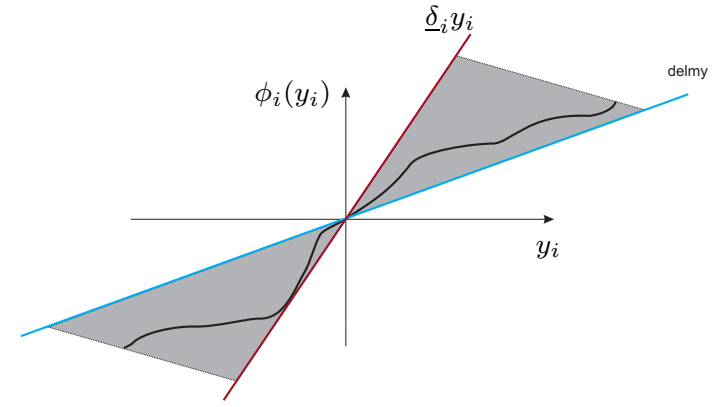

Fig. 1. Sector-bounded nonlinearity.

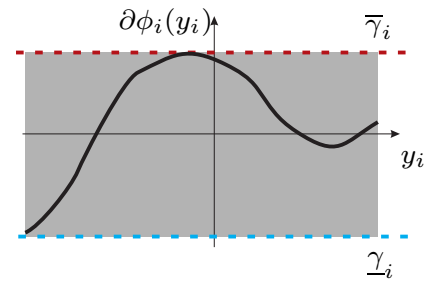

Fig. 2. Bounds on the nonlinearity slope.

For the particular case of $\mathcal{X}_{0}=\mathbb{R}^{n}$ a solution to the above problem gives a certificate of the global stability of the origin.

\section{Preliminary Results}

In this section we recall sector inequalities related to slope-restricted nonlinearities and provide conditions for the positive definiteness of the following quadratic-like expressions

$$
\begin{aligned}
m(x, \phi) & =\left[\begin{array}{l}
x \\
\phi
\end{array}\right]^{T} M\left[\begin{array}{l}
x \\
\phi
\end{array}\right], \\
n(x, \phi, \dot{\phi}) & =\left[\begin{array}{l}
x \\
\phi \\
\dot{\phi}
\end{array}\right]^{T} N\left[\begin{array}{l}
x \\
\phi \\
\dot{\phi}
\end{array}\right]
\end{aligned}
$$

with $M \in \mathbb{R}^{(n+m) \times(n+m)}$ and $N \in \mathbb{R}^{(n+2 m) \times(n+2 m)}$. We assume that $\phi$ satisfies (2) and that $\dot{\phi}$ denotes the timederivative of the nonlinearity $\phi$ along the trajectories of (1).

Lemma 3.1: Assume $\phi$ satisfies (2a)-(2b). The inequalities

$$
\left(\phi_{i}\left(y_{i}\right)-\underline{\delta}_{i} y_{i}\right)\left(\bar{\delta}_{i} y_{i}-\phi_{i}\left(y_{i}\right)\right) \geq 0
$$

$i=1, \ldots, m$ hold for $y \in \mathcal{Y}_{0}$.

The proof of the above lemma is straightforwardly obtained from $(2 \mathrm{~b})$. The following lemma presents an inequality related to the slope restrictions on $\phi$.

Lemma 3.2: Assume $\phi$ satisfies (2a)-(2c) and that Assumption 2.1 holds. The inequalities

$$
\left(\dot{\phi}_{i}\left(y_{i}\right)-\underline{\gamma}_{i} \dot{y}_{i}\right)\left(\bar{\gamma}_{i} \dot{y}_{i}-\dot{\phi}_{i}\left(y_{i}\right)\right) \geq 0
$$

$i=1, \ldots, m$ hold for $y \in \mathcal{Y}_{0}$.

Proof: From (2c) we have

$$
0 \leq\left(\partial \phi_{i}-\underline{\gamma}_{i}\right)\left(\bar{\gamma}_{i}-\partial \phi_{i}\right) .
$$


Multiplying the above expression by $\dot{y}^{2}$, with $\dot{y}$ the timederivative of $y$ along the trajectories of (1), gives

$$
\begin{aligned}
0 & \leq \dot{y}_{i}^{2}\left(\partial \phi_{i}-\underline{\gamma}_{i}\right)\left(\bar{\gamma}_{i}-\partial \phi_{i}\right) \\
& =\left(\partial \phi_{i} \dot{y}_{i}-\underline{\gamma}_{i} \dot{y}_{i}\right)\left(\bar{\gamma}_{i} \dot{y}_{i}-\partial \phi_{i} \dot{y}_{i}\right) \\
& =\left(\dot{\phi}_{i}\left(y_{i}\right)-\underline{\gamma}_{i} \dot{y}_{i}\right)\left(\bar{\gamma}_{i} \dot{y}_{i}-\dot{\phi}_{i}\left(y_{i}\right)\right) .
\end{aligned}
$$

Note that, under Assumption 2.1, we have $y(x, \phi)=$ $C x+D \phi$, and $\dot{y}_{i}(x, \phi, \dot{\phi})=C A x+C B \phi+D \dot{\phi}$. It follows that (5) and (6), with $y_{i}$ being the $i$ th row of $y$ satisfying (1), are quadratic on $x, \phi$ and $\dot{\phi}$. The following lemmas use Lemmas 3.1 and 3.2 to formulate conditions for the positivity of the expressions (4).

Lemma 3.3: If there exists a matrix $T \in \mathbb{D}_{\geq 0}^{m}$ such that

$$
m(x, \phi)-s_{1}(T, x, \phi)>0
$$

with

$$
s_{1}(T, x, \phi):=(\phi-\underline{\Delta} y(x)) T(\bar{\Delta} y(x)-\phi)
$$

then $m(x, \phi)>0$ for all $x \in \mathbb{R}^{n}$ and for any $\phi: \mathbb{R}^{m} \rightarrow \mathbb{R}^{m}$ satisfying $(2 \mathrm{a})-(2 \mathrm{~b})$.

Lemma 3.4: If there exists matrices $T_{1}, T_{2} \in \mathbb{D}_{\geq 0}^{m}$ such that

$$
n(x, \phi, \dot{\phi})-s_{1}\left(T_{1}, x, \phi\right)-s_{2}\left(T_{2}, x, \phi, \dot{\phi}\right)>0
$$

with $s_{1}$ defined in $(7 \mathrm{~b})$ and

$$
s_{2}\left(T_{2}, x, \phi, \dot{\phi}\right):=(\dot{\phi}-\underline{\Gamma} \dot{y}(x, \phi, \dot{\phi})) T_{2}(\bar{\Gamma} \dot{y}(x, \phi, \dot{\phi})-\dot{\phi})
$$

then $n(x, \phi, \dot{\phi})>0$ for all $x \in \mathbb{R}^{n}$ and for all $\phi: \mathbb{R}^{m} \rightarrow$ $\mathbb{R}^{m}$ satisfying (2).

Proof: If (8a) holds we have

$$
\begin{aligned}
n(x, \phi, \dot{\phi})> & s_{1}\left(T_{1}, x, \phi\right)+s_{2}\left(T_{2}, x, \phi, \dot{\phi}\right) \\
= & \sum_{i=1}^{m}\left[T_{1(i, i)}\left(\phi_{i}-\underline{\delta}_{i} y_{i}(x)\right)\left(\bar{\delta}_{i} y_{i}(x)-\phi_{i}\right)\right. \\
& \left.\quad+T_{2(i, i)}\left(\dot{\phi}_{i}-\underline{\gamma}_{i} \dot{y}_{i}(x)\right)\left(\bar{\gamma}_{i} \dot{y}_{i}(x)-\dot{\phi}_{i}\right)\right] \\
\geq & 0
\end{aligned}
$$

where we have used inequalities $(2 \mathrm{~b})$ and $(2 \mathrm{c})$ and the non-negative terms $T_{j(i, i)} \geq 0, j=1,2, i=1, \ldots, m$.

From the above lemmas, we note that the nonnegativity of $m(x, \phi)$ and $n(x, \phi, \dot{\phi})$ does not necessarily require the positive-semidefiniteness of matrices $M$ and $N$ in (4). This stems from the fact that $x, \phi$ and $\dot{\phi}$ are not independent but are related via the inequalities $s_{1}(x, \phi) \geq 0$ and $s_{2}(x, \phi, \dot{\phi}) \geq 0$, that hold on the set $\mathcal{X}_{0}$, following the definition of the sector inequalities $(2 \mathrm{~b}),(2 \mathrm{c})$ and the definition of the set $\mathcal{X}_{0}$. The inequality (7a) has been extensively used to verify the negative-definiteness of the derivative of the LF in the stability analysis of input saturating systems [10, page 232] (see also [8, 17] for local sector conditions). In [18, 5], (8a) has been used to study the time-derivative of the LFs with terms (4a). In the following section, the stability analysis of system (1) with LF containing terms of the form given in (4a) uses
Lemma 3.3 to formulate conditions for the positivity of the LF itself (not only to study the time-derivative of the $\mathrm{LF})$.

\section{MAIN RESULTS}

This section is concerned with the LF of the form

$$
\begin{gathered}
V(x)=V_{0}(x)+\sum_{i=1}^{m} \lambda_{i} \int_{0}^{y_{i}(x)} \phi_{i}(s) d s \\
V_{0}(x)=\left[\begin{array}{c}
x \\
\phi(y(x))
\end{array}\right]^{T}\left[\begin{array}{ll}
P_{11} & P_{12} \\
P_{12}^{T} & P_{22}
\end{array}\right]\left[\begin{array}{c}
x \\
\phi(y(x))
\end{array}\right]
\end{gathered}
$$

The terms involving the integral are referred to as the Lur'e terms [2].

The positivity of the above function can be enforced by requiring both $P:=\left[\begin{array}{ll}P_{11} & P_{12} \\ P_{12}^{T} & P_{22}\end{array}\right]>0$ and $\lambda_{i} \geq 0$, as discussed in [18] and as in [19] for the case $P_{12}=0, P_{22}=$ 0 . For the latter structure, the relaxation of the nonnegativity of the coefficients $\lambda_{i}$ was considered in [14], [6] and [1].

Define $\Lambda:=\operatorname{diag}\left(\lambda_{1}, \ldots, \lambda_{m}\right)$ to be used in the following lemma that presents conditions for the positivity of $V$ without imposing positive-definiteness of $P$ nor the non-negativity of the coefficients $\lambda_{i}$.

Lemma 4.1: Consider $V$ as in (9) with $\phi$ satisfying (2a)-(2b) and such that Assumption 2.1 holds. If there exists a matrix $\tilde{\Lambda} \in \mathbb{D}_{\geq 0}^{m}$ such that

$$
\begin{aligned}
& \Lambda \geq-\tilde{\Lambda} \\
& V_{0}(x)-\frac{1}{2} y^{T}(x)(\bar{\Delta}-\underline{\Delta}) \tilde{\Lambda} y(x)>0,
\end{aligned}
$$

then $V(x)>0 \forall x \in \mathcal{X} \subset \mathbb{R}^{n}$

Proof: We prove the claim with the relations in (11) (see the top of the next page), where we use (10a) to obtain a positive-definite lower bound for (9) and we assume well-posedness of the algebraic loop for all $x \in \mathcal{X}$.

Remark 4.1: [Lur'e terms in the LF of [18]] Consider the function

$$
\bar{V}(x)=\left[\begin{array}{c}
x \\
\phi(x)
\end{array}\right]^{T} \bar{P}\left[\begin{array}{c}
x \\
\phi(x)
\end{array}\right]+\sum_{j=1}^{4} \sum_{i=1}^{m} g_{j, i}(x)
$$

where

$$
\begin{aligned}
& g_{1, i}(x):=\mu_{1, i} \int_{0}^{y_{i}(x)} \phi_{i}(s) \mathrm{d} s \\
& g_{2, i}(x):=\mu_{2, i} \int_{0}^{y_{i}(x)}\left[\bar{\delta}_{i} s-\phi_{i}(s)\right] \mathrm{d} s \\
& g_{3, i}(x):=\mu_{3, i} \int_{0}^{y_{i}(x)}\left[\bar{\gamma}_{i}-\partial \phi_{i}(s)\right] s \mathrm{~d} s \\
& g_{4, i}(x):=\mu_{4, i} \int_{0}^{y_{i}(x)} \partial \phi_{i}(s)\left[\bar{\delta}_{i} s-\phi_{i}(s)\right] \mathrm{d} s
\end{aligned}
$$

with $\bar{P}>0$ and $\mu_{j, i} \geq 0, i=1, \ldots, m, j=1, \ldots, 4$ which clearly yield $g_{j, i}(x) \geq 0 i=1, \ldots, m, j=1, \ldots, 4$ for $\phi$ satisfying (2) with $\underline{\delta}_{i}=\underline{\gamma}_{i}=0, i=1, \ldots, m$. In [18], $\bar{V}$ 


$$
\begin{aligned}
& V(x)=V_{0}(x)+\sum_{i=1}^{m} \lambda_{i} \int_{0}^{y_{i}(x)} \phi_{i}(s) d s \\
& \geq V_{0}(x)+\sum_{i=1}^{m} \lambda_{i} \int_{0}^{y_{i}(x)}\left(\phi_{i}(s)-\underline{\delta}_{i} s\right) d s \\
& \geq V_{0}(x)-\sum_{i=1}^{m} \tilde{\lambda}_{i} \int_{0}^{y_{i}(x)}\left(\phi_{i}(s)-\underline{\delta}_{i} s\right) d s \\
& =V_{0}(x)-\frac{1}{2} y^{T}(x)(\bar{\Delta}-\bar{\Delta}) \tilde{\Lambda} y(x)-\sum_{i=1}^{m} \tilde{\lambda}_{i} \int_{0}^{y_{i}(x)}\left(\phi_{i}(s)-\underline{\delta}_{i} s\right) d s \\
& =V_{0}(x)-\frac{1}{2} y^{T}(x)(\bar{\Delta}-\bar{\Delta}-\underline{\Delta}) \tilde{\Lambda} y(x)-\sum_{i=1}^{m} \tilde{\lambda}_{i} \int_{0}^{y_{i}(x)} \phi_{i}(s) d s \\
& =V_{0}(x)-\frac{1}{2} y^{T}(x)(\bar{\Delta}-\underline{\Delta}) \tilde{\Lambda} y(x)+\sum_{i=1}^{m} \tilde{\lambda}_{i} \int_{0}^{y_{i}(x)}\left(\left(\bar{\delta}_{i} s-\phi_{i}(s)\right) d s\right. \\
& =\underbrace{V_{0}(x)-\frac{1}{2} y^{T}(x)(\bar{\Delta}-\underline{\Delta}) \tilde{\Lambda} y(x)}_{>0 \text { from }(10 \mathrm{~b})}+\underbrace{\sum_{i=1}^{m} \tilde{\lambda}_{i} \int_{0}^{y_{i}(x)}\left(\bar{\delta}_{i} s-\phi_{i}(s)\right) d s}_{\geq 0 \text { from } \tilde{\Lambda} \geq 0 \text { and }(2 \mathrm{~b}) .}
\end{aligned}
$$

was used to obtain upper-bounds of the induced $\mathcal{L}_{2}$ gain for system (1) with additive disturbance terms.

By using the relations

$$
\begin{aligned}
& \int_{0}^{y_{i}} \phi_{i}(s) \partial \phi_{i}(s) \mathrm{d} s=\frac{1}{2} \phi_{i}^{2}\left(y_{i}\right) \\
& \int_{0}^{y_{i}} \partial \phi_{i}(s) s \mathrm{~d} s=\phi_{i}^{2}\left(u_{i}\right) u_{i}+\int_{0}^{y_{i}} \phi_{i}(s) \mathrm{d} s
\end{aligned}
$$

it is straightforward to obtain

$$
\begin{aligned}
\sum_{j=1}^{4} \sum_{i=1}^{m} g_{j, i}(x)= & {\left[\begin{array}{c}
x \\
\phi(x)
\end{array}\right]^{T} \bar{M}\left[\begin{array}{c}
x \\
\phi(x)
\end{array}\right] } \\
& +\sum_{i=1}^{m} \mu_{i} \int_{0}^{y_{i}(x)} \phi_{i}(s) \mathrm{d} s
\end{aligned}
$$

with $\bar{M}=\left[\begin{array}{ll}C^{T} & 0 \\ D^{T} & I\end{array}\right] M\left[\begin{array}{ll}C & D \\ 0 & I\end{array}\right]$ where

$$
M=\left[\begin{array}{cc}
\bar{\Delta} M_{2}+\bar{\Gamma} M_{3} & \frac{1}{2}\left(\bar{\Delta} M_{4}-M_{3}\right) \\
\frac{1}{2}\left(\bar{\Delta} M_{4}-M_{3}\right) & -\frac{1}{2} M_{4}
\end{array}\right],
$$

with $M_{j}=\operatorname{diag}\left(\mu_{j, 1}, \ldots, \mu_{j, m}\right), j=1, \ldots, 4$, and $\mu_{i}=$ $\left(\mu_{1, i}-\mu_{2, i}+\mu_{3, i}-\bar{\delta}_{i} \mu_{4, i}\right)$.

The above expressions show that (12) can be written as (9a) with matrix $P$ replaced by matrix $\bar{P}+\bar{M}$. However, since matrix $\bar{P}+\bar{M}$ is not necessarily positive definite and the coefficients $\mu_{i}$ are not sign definite (as can be seen from the above expressions: note the $-\frac{1}{2} M_{4}$ term in the $(2,2)$ block of matrix $M)$, the results of [18] imply that $V$ as defined in (9a) may have the term $V_{0}$ with $P$ which is not positive definite and Lur'e terms that are not necessarily non-negative.

Here, instead of using $\bar{V}$ with positive-definite and non-negative $\mu_{i, j}$ we will apply the conditions for the positivity of $V$ as detailed in Lemma 4.1.

The following theorem presents conditions for the stability of the origin of Lur'e system (1) with sloperestricted nonlinearities:

Theorem 4.1: If there exists a matrix $P \in \mathbb{R}^{n+m \times n+m}$, matrices $\Lambda \in \mathbb{D}^{m}, \tilde{\Lambda}, T_{0}, T_{1}, T_{2} \in \mathbb{D}_{\geq 0}^{m}$ and a scalar $\alpha>0$ such that (10a) holds,

$$
V_{0}(x)-\frac{1}{2} y^{T}(\bar{\Delta}-\underline{\Delta}) \tilde{\Lambda} y-s_{1}\left(T_{0}, x, \phi\right)>0
$$

$\forall x \in \mathbb{R}^{n}, \phi \in \mathbb{R}^{m}$,

$$
\begin{aligned}
-\left\langle\left[\begin{array}{c}
\nabla_{x} V \\
\nabla_{\phi} V
\end{array}\right],\left[\begin{array}{c}
A x+B \phi \\
\dot{\phi}
\end{array}\right]\right\rangle \\
\quad-s_{1}\left(T_{1}, x, \phi\right)-s_{2}\left(T_{2}, x, \phi, \dot{\phi}\right)>0
\end{aligned}
$$

$\forall x \in \mathbb{R}^{n}, \phi \in \mathbb{R}^{m}$, and

$$
\mathcal{E}(V, \alpha) \subseteq \mathcal{X}_{0}
$$

then the origin of (1) is locally asymptotically stable and $\mathcal{E}(V, \alpha)$ is an estimate of its region of attraction. In the case $\mathcal{X}_{0}=\mathbb{R}^{n}$, then the origin is globally asymptotically stable.

Proof: Provided the sector inequalities (2b) and (2c) hold, that is, provided the trajectories live in the set $\mathcal{X}_{0}$, it follows from lemmas 3.3- 3.4that $V(x)$ is positive and $\dot{V}(x)=\left\langle\left[\begin{array}{c}\nabla_{x} V \\ \nabla_{\phi} V\end{array}\right],\left[\begin{array}{c}A x+B \phi \\ \dot{\phi}\end{array}\right]\right\rangle$, the time-derivative of $V$ is negative along the trajectories of system (1). Following [10, Theorem 4.1], if (15c) holds, the $\mathcal{E}(V, \alpha)$ is invariant and contractive, hence defining an estimate of the basin of attraction of (1).

A convenient property of the quadratic inequalities (15) is that their representation is affine on $P, \Lambda, \tilde{\Lambda}, T_{i}$, $i=\{0,1,2\}$. These matrices can be variables of a convex semi-definite program whenever the system matrices $(A, B, C, D)$ and the sector bounds $\underline{\Delta}, \bar{\Delta}, \underline{\Gamma}, \bar{\Gamma}$ are given.

The inclusion condition (15c) may, in some cases, be specified in terms of quadratic-like inequalities as in the Lyapunov inequalities. This depends on the description of the set $\mathcal{X}_{0}$ and on the computation of an explicit form for the Lur'e term. In Section V, Example 5.2 introduces a set $\mathcal{X}_{0}$ defined by linear inequalities and a logarithmic nonlinearity which allows for a quadratic-like representation of the LF.

\section{Numerical Results}

We present numerical results obtained with the solution to the linear matrix inequalities associated to the inequalities in Theorem 4.1.

Example 5.1: This example considers the SISO system $G(s)=\frac{-0.5 s^{2}-s+1}{\left(s^{2}+0.1 s+1\right)}$, with the bounds of $\phi$ given by $\underline{\delta}=0$, $\underline{\gamma}=0, \bar{\delta}=\delta^{*}, \bar{\gamma}=\delta^{*}$. With a bisection algorithm 
we maximize $\delta^{*}$ such that the inequalities (15) hold. In order to compare with different LF structures from the literature we also compute the maximal value of $\delta^{*}$ such that the inequalities (15) hold for $V$, for $V_{0}$ (i.e. $V$ with $\Lambda=0$ ), for the LF of the Circle Criterion, $V_{Q}(x)=x^{T} P_{11} x$, which corresponds to $V$ with $P_{12}=$ $0, P_{22}=0, \Lambda=0$ and for the LF of the Popov criterion

$$
V_{Q L}(x)=x^{T} P_{11} x+\sum_{i=1}^{m} \lambda_{i} \int_{0}^{y_{i}} \phi_{s}(s) \mathrm{d} s,
$$

which corresponds to $V$ with $P_{12}=0, P_{22}=0$. The obtained results are detailed in Table I where it is shown that with the LF defined by $V$ we obtain the least conservative upper bound on the sector condition $\delta^{*}$.

\begin{tabular}{|c|c|c|c|c|}
\hline & $V_{Q}$ & $V_{0}$ & $V_{Q L}$ & $V$ \\
\hline$\delta^{*}$ & 0.236 & 0.236 & 0.725 & 0.999 \\
\hline
\end{tabular}

Maximum Value of $\delta^{*}$ For Different structures of The LF.

The function $V$ obtained for system (1) with the bounds defined by $\delta^{*}$ corresponds to the following values for $P$ and $\Lambda$

$$
P=\left[\begin{array}{ccc}
2.3833 & -0.0149 & 1.4652 \\
-0.0149 & 2.3222 & -0.2928 \\
1.4652 & -0.2928 & 1.3280
\end{array}\right], \Lambda=-2.5254
$$

For the function $V_{Q L}$ we obtain $P_{11}=\left[\begin{array}{ll}1.7996 & 0.1182 \\ 0.1182 & 1.9164\end{array}\right], \Lambda=$ -2.7677 . Note that the Lur'e coefficients are negative. For the function $V_{0}$ we obtain

$$
P=\left[\begin{array}{ccc}
0.5148 & 0.0221 & 0.0941 \\
0.0221 & 0.5325 & -0.1255 \\
0.0941 & -0.1255 & -0.5802
\end{array}\right]
$$

where the corresponding $P_{22}$ block is negative (entry $-0.5802)$. And for $V_{Q}$ we have computed

$$
P_{11}=\left[\begin{array}{ll}
0.5323 & 0.0415 \\
0.0415 & 0.5325
\end{array}\right] \text {. }
$$

Example 5.2: Motivated by applications of electrical energy storage devices known as supercapacitors [4] we now study a SISO system with the nonlinearity $\phi(y)=$ $\ln (1+y)$, the shifted logarithmic function as depicted in Figure 3. This nonlinearity has an asymptote at $y_{i}=-1$ and is defined in $\mathcal{Y}=(-1, \infty)$.

Set $\mathcal{Y}_{0}=[\underline{y}, \bar{y}]$, with $-1<\underline{y}<0,0<\bar{y}<\infty$. The corresponding sector and slope bounds $\underline{\delta}, \bar{\delta}, \underline{\gamma}, \bar{\gamma}$ in $(2 \mathrm{~b}),(2 \mathrm{c})$ are given by

$$
\begin{array}{ll}
\underline{\delta}=\frac{\ln (1+\bar{y})}{\bar{y}}, & \bar{\delta}=\frac{\ln (1+\underline{y})}{\underline{y}}, \\
\underline{\gamma}=\frac{1}{1+\bar{y}}, & \bar{\gamma}=\frac{1}{1+\underline{y}} .
\end{array}
$$

We take $\underline{y}=-0.99$ and $\bar{y}=2$ and use the corresponding bounds (16) to perform the local analysis. We consider the SISO system $G(s)=\frac{0.1(s+0.1)}{\left(s^{2}+10 s+1\right)}$ and compute

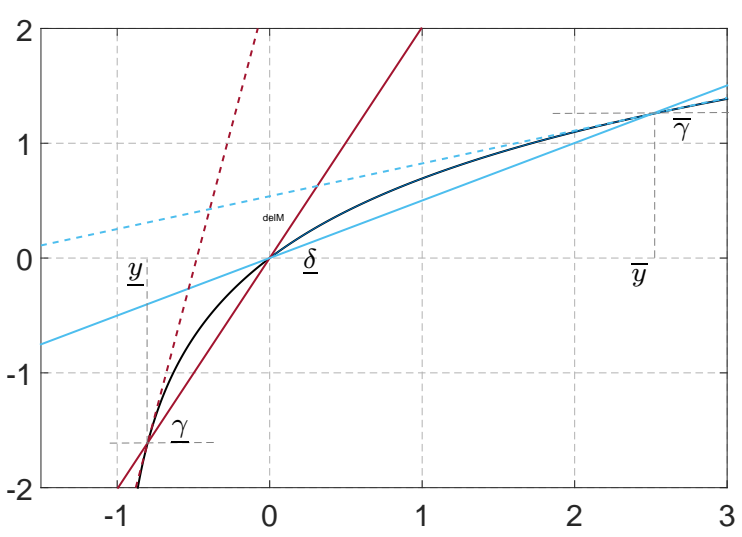

Fig. 3. Local sector bounds and slope bounds for the function $\phi(y)=\ln (1+y)$ (black solid lines). Lines crossing the origin define the sector $\underline{f}(y):=\underline{\delta} y$ (blue solid line), $\bar{f}(y):=\bar{\delta} y$ (red solid line), lines depicting the slope restriction, passing through $\bar{y}$, $\bar{g}(y):=\ln (1+\bar{y})+\underline{\gamma} y-\underline{\gamma} \bar{y}$ (blue dashed line), and through $\underline{y}$, $\underline{g}(y):=\ln (1+\underline{y})+\bar{\gamma} \bar{y}-\bar{\gamma} \underline{y}$ (red dashed line).

the largest level sets of the Lyapunov functions $V, V_{0}, V_{Q}$, $V_{Q L}$ that satisfy the inclusion. The inclusion was checked by inspection and we do not present inclusion conditions. These level sets are shown in figure 4, where trajectories that asymptotically converge to the origin are depicted in green. Note that by including more information about the nonlinearity, the function $V$ gives a better description of the domain of attraction when compared with the estimates obtained with $V_{Q}, V_{Q L}$ and $V_{0}$.

For this example, the computed values of $P$ and $\Lambda$ that formed $V$ were

$$
P=\left[\begin{array}{ccc}
0.0808 & -0.0343 & 0.4712 \\
-0.0343 & 1.6648 & -0.0093 \\
0.4712 & -0.0093 & -0.0106
\end{array}\right], \Lambda=-0.2914
$$

showing that both the positive definite requirement on $P$ and the non-negative condition on $\Lambda$ could simultaneously be relaxed.

For the logarithmic nonlinearity, the Lur'e terms are given by

$$
\int_{0}^{y_{i}} \ln (1+s) \mathrm{d} s=\left(1+y_{i}\right) \ln \left(1+y_{i}\right)-y_{i}
$$

hence, for $\phi(y(x))=\left[\ln \left(1+y_{1}(x)\right) \ldots \ln \left(1+y_{m}(x)\right)\right]$, the function $V(x)$ in (9a) has quadratic form.

\section{Conclusions}

In this paper, stability analysis of Lur'e type systems with slope-restricted nonlinearities was carried out for LFs that have a quadratic-like term on the state and the nonlinearity and Lur'e type terms. We have proposed relaxed conditions for the positivity of the LF (cf. Lemma 4.1) and have used sector inequalities to propose conditions for the global and local stability of the origin of Lur'e systems. 


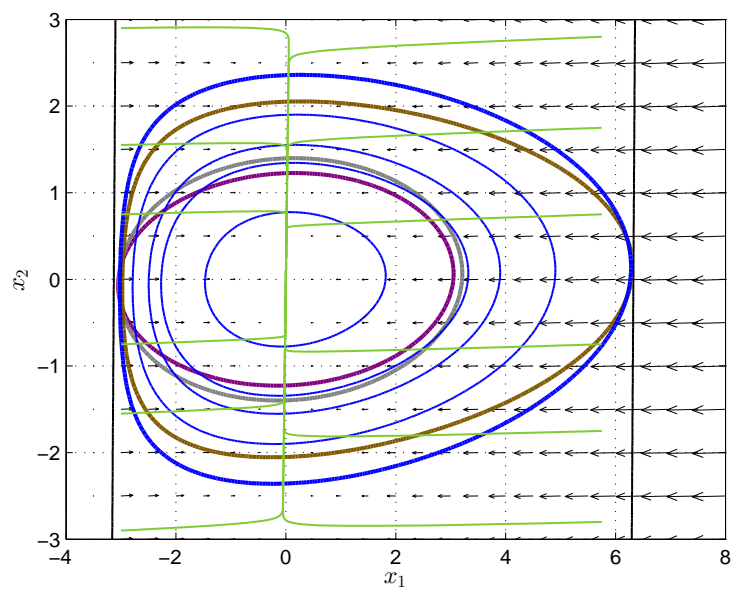

Fig. 4. Level sets of the LF $V$ (blue solid lines), the largest level sets contained in $\mathcal{X}_{0}$ of $V_{0}$ (orange solid lines), of $V_{Q L}$ (grey solid lines), and of $V_{Q}$ (purple solid lines), a set of stable trajectories (green solid lines) and the set $\mathcal{X}_{0}$, in which the local sector condition holds (delimited by black solid lines).

The inequalities on the proposed conditions are of quadratic nature and are affine in the Lyapunov function coefficients and in the multipliers associated with the sector inequalities. Thanks to these properties, it is possible to obtain equivalent LMI formulations.

Numerical examples illustrate the results and provide comparison between them to different LF structures. For a SISO Lur'e system of order two defined by a logarithmic nonlinearity, the obtained estimates of the region of attraction significantly improve estimates defined by quadratic functions.

We are currently developing conditions for statefeedback design for Lur'e systems using the Lyapunov functions studied in this paper.

\section{REFERENCES}

[1] M.A. Aizerman and F.R. Gantmacher. Absolute stability of regulator systems. Holden-Day, 1964.

[2] S.P. Boyd, L. El Ghaoui, E. Feron, and V. Balakrishnan. Linear matrix inequalities in system and control theory, volume 15. SIAM, 1994.

[3] J. Carrasco, M. C. Turner, and W. P. Heath. Zames-Falb multipliers for absolute stability: From O'Shea's contribution to convex searches. In Proceedings of the 14th European Control Conference, pages 1261-1278, 2015.

[4] R. Drummond, D.A. Howey, and S.R. Duncan. Loworder mathematical modelling of electric double layer supercapacitors using spectral methods. Journal of Power Sources, 277:317-328, 2015.

[5] A. Garulli, A. Masi, G. Valmorbida, and L. Zaccarian. Global stability and finite 12m-gain of saturated uncertain systems via piecewise polyno- mial lyapunov functions. IEEE Transactions on Automatic Control, 58(1):242-246, Jan 2013.

[6] W.P. Heath and G. Li. Lyapunov functions for the multivariable Popov criterion with indefinite multipliers. Automatica, 45(12):2977-2981, 2009.

[7] T. Hu and Z. Lin. Composite quadratic Lyapunov functions for constrained control systems. IEEE Transactions on Automatic Control, 48(3):440-450, 2003.

[8] T. Hu, A.R. Teel, and L. Zaccarian. Stability and performance for saturated systems via quadratic and nonquadratic Lyapunov functions. IEEE Transactions on Automatic Control,, 51(11):1770-1786, 2006.

[9] R.E. Kalman. Physical and mathematical mechanisms of instability in nonlinear automatic control systems. Transactions of the ASME, 79(3):553-566, 1957.

[10] H. K. Khalil. Nonlinear systems, volume 3. Prentice hall Upper Saddle River, NJ, 2002.

[11] G.A. Leonov, V. Ponomarenko, and V.B. Smirnova. Frequency-domain methods for nonlinear analysis: theory and applications, volume 9. World Scientific, 1996.

[12] A. Megretski and A. Rantzer. System analysis via integral quadratic constraints. IEEE Transactions on Automatic Control, 42(6):819-830, 1997.

[13] R.E. O'Shea. An improved frequency-time domain stability criterion for autonomous continuous systems. IEEE Transactions on Automatic Control, 12(6):725-731, 1967.

[14] P. Park. A revisited Popov criterion for nonlinear Lur'e systems with sector-restrictions. International Journal of Control, 68(3):461-470, 1997.

[15] P. Park. Stability criteria of sector-and sloperestricted Lur'e systems. IEEE Transactions on Automatic Control, 47(2):308-313, 2002.

[16] G. Schmitt. Frequency domain evaluation of circle criterion, Popov criterion and off-axis circle criterion in the MIMO case. International Journal of Control, 72(14):1299-1309, 1999.

[17] S. Tarbouriech, C. Prieur, and J. M. Gomes da Silva Jr. Stability analysis and stabilization of systems presenting nested saturation. IEEE Transactions on Automatic Control, 51(8):1364-1371, 2006.

[18] M. C. Turner and M. Kerr. Lyapunov functions and $\mathcal{L}_{2}$ gain bounds for systems with slope restricted nonlinearities. Systems \& Control Letters, 69:1-6, 2014.

[19] M. Vidyasagar. Nonlinear systems analysis, volume 42. SIAM, 2002.

[20] G. Zames and P.L. Falb. Stability conditions for systems with monotone and slope-restricted nonlinearities. SIAM Journal on Control, 6(1):89-108, 1968 . 